\title{
Role of endothelial Nox2 NADPH oxidase in angiotensin II-induced hypertension and vasomotor dysfunction
}

\author{
Colin E. Murdoch · Sara P. Alom-Ruiz • \\ Minshu Wang $\cdot$ Min Zhang $\cdot$ Simon Walker $\cdot$ \\ Bin Yu • Alison Brewer • Ajay M. Shah
}

Received: 27 October 2010/Revised: 17 March 2011/Accepted: 11 April 2011/Published online: 29 April 2011

(C) The Author(s) 2011. This article is published with open access at Springerlink.com

\begin{abstract}
NADPH oxidase (Nox)-derived reactive oxygen species (ROS) are known to be involved in angiotensin II-induced hypertension and endothelial dysfunction. Several Nox isoforms are expressed in the vessel wall, among which Nox 2 is especially abundant in the endothelium. Endothelial Nox2 levels rise during hypertension but little is known about the cell-specific role of endothelial Nox2 in vivo. To address this question, we generated transgenic mice with endothelial-specific overexpression of Nox2 (Tg) and studied the effects on endothelial function and blood pressure. $\mathrm{Tg}$ had an about twofold increase in endothelial Nox2 levels which was accompanied by an increase in p22phox levels but no change in levels of other Nox isoforms or endothelial nitric oxide synthase (eNOS). Basal NADPH oxidase activity, endothelial function and blood pressure were unaltered in Tg compared to wild-type littermates. Angiotensin II caused a greater increase in ROS production in Tg compared to wild-type aorta and attenuated acetylcholine-induced vasorelaxation. Both low and high dose chronic angiotensin II infusion increased
\end{abstract}

Electronic supplementary material The online version of this article (doi:10.1007/s00395-011-0179-7) contains supplementary material, which is available to authorized users.

C. E. Murdoch · S. P. Alom-Ruiz · M. Wang · M. Zhang ·

S. Walker - B. Yu - A. Brewer - A. M. Shah

Cardiovascular Division, King's College London British Heart

Foundation Centre, London SE5 9PJ, UK

A. M. Shah $(\square)$

Cardiovascular Division, The James Black Centre,

King's College London, 125 Coldharbour Lane,

London SE5 9NU, UK

e-mail: ajay.shah@kcl.ac.uk telemetric ambulatory blood pressure more in Tg compared to wild-type, but with different patterns of BP change and aortic remodeling depending upon the dose of angiotensin II dose. These results indicate that an increase in endothelial Nox2 levels contributes to angiotensin II-induced endothelial dysfunction, vascular remodeling and hypertension.

Keywords Endothelium - Vascular tone - NADPH oxidase $\cdot$ Hypertension $\cdot$ Reactive oxygen species

\section{Introduction}

Increased production of reactive oxygen species (ROS) is involved in the pathophysiology of cardiovascular diseases such as hypertension, especially in the setting of increased activation of the renin-angiotensin system $[7,14,21]$. Increased levels of vascular ROS may induce vascular endothelial dysfunction through the superoxide-mediated inactivation of endothelium-derived vasodilator nitric oxide (NO) as well as modulate intracellular signaling pathways that promote vascular remodeling [21]. Both these mechanisms can contribute to the development of angiotensin-dependent hypertension although redox-regulated pathways in the kidneys and the central nervous system are also important.

NADPH oxidase proteins are major sources of ROS [2, $4,18]$. All NADPH oxidases contain a core subunit, termed Nox, which catalyzes the transfer of electrons from NADPH to molecular $\mathrm{O}_{2}$ and leads to ROS generation. Five Nox isoforms (Nox1-5) have been identified, which form the basis of distinct NADPH oxidases and have varying requirements for other protein subunits. Among these isoforms, Nox5 is absent in rodents [2]. In the vasculature, Nox 2 and Nox4 are expressed in the endothelium, 
whereas vascular smooth muscle cells (VSMC) contain predominately Nox4 and Nox1 [2, 4]. Nox2 is also expressed in adventitial fibroblasts and in human conduit artery VSMC [2, 4]. There are significant biochemical differences between Nox1, 2 and 4. Whilst each of the three Noxs (i.e. Nox1, Nox 2 and Nox4) forms a heterodimer with a p22phox subunit, Nox1 and 2 require the binding of additional regulatory subunits for their activation whereas Nox 4 is constitutively active and does not require other cytosolic subunits for its activity. Nox2 activation is induced by agonists such as angiotensin II, which cause post-translational modifications of oxidase regulatory subunits (p47phox, p67phox, p40phox and Rac1) and their subsequent association with the Nox2p22phox heterodimer. The isoform-specific expression of Noxs in the different cell types of the vessel wall and the co-expression of more than one isoform in individual cell types both suggest that the different isoforms may have distinct roles. However, cell-specific roles of Nox isoforms in vivo remain poorly understood.

It is well established that NADPH oxidases are involved in the pathophysiology of angiotensin II-dependent hypertension and endothelial dysfunction but the roles of the different Nox isoforms is less clear [4, 7, 17, 27]. Nox 1 knockout mice were reported to have a reduced basal blood pressure and an attenuation of angiotensin II-induced hypertension and endothelial dysfunction $[11,20]$, whereas mice with VSMC-targeted overexpression of Nox1 had vascular hypertrophy and an enhanced pressor response to angiotensin II [8]. Transgenic mice with VSMC-specific overexpression of Rac1 (which is required for both Nox1 and Nox2 activity) also showed enhanced angiotensin IIinduced hypertension [13]. Studies in tissues from human patients with hypertension, diabetes, heart failure or aging have correlated endothelial vasodilator dysfunction with increased Nox2 expression and activity [9, 10]. Nox2 knockout mice show improved endothelial dysfunction in a model of renovascular hypertension [17] but no change in basal blood pressure [5] or the hypertensive response to chronic angiotensin II infusion [16], nor to chronic activation of the renin-angiotensin system [28]. The above studies suggest that VSMC Nox1 is important in angiotensin II-induced hypertension but the cell-specific roles of Nox 2 and, in particular, the contribution of endothelial Nox2 remains unclear.

In this study, we generated transgenic mice with endothelium-targeted overexpression of Nox 2 and investigated the effect on vascular function and angiotensin II-induced hypertension. We report that increases in endothelial levels of Nox 2 in vivo contribute significantly to the enhancement of angiotensin II-dependent hypertension, vascular remodeling and endothelial dysfunction.

\section{Methods}

Transgenic mice and tissues

All animal procedures were conducted in accordance with the Guidance on the Operation of the Animals (Scientific Procedures) Act, 1986 (UK Home Office). Transgenic mice with endothelial-targeted overexpression of Nox2 were generated utilizing a tie 2 promoter/enhancer construct [26] containing the full length human Nox 2 cDNA sequence. Fertilized oocytes from CBA/C57B16 mice were microinjected with the construct and founder progeny was identified by PCR analysis of genomic DNA. Two independent transgenic lines were generated and backcrossed for $>10$ generations into a C57B16 background. Experiments were conducted using heterozygous male mice and wild-type littermate controls aged between 8 and 16 weeks. Aortic tissues for immunoblotting or mRNA expression analyses were snap frozen in liquid nitrogen and stored at $-80^{\circ} \mathrm{C}$. Endothelial denudation on aortic rings was performed using a fine wire threaded into the lumen.

\section{Surgical procedures}

Telemeter transducers (PA-C10, Data Sciences International, St. Paul, MN) were implanted under $2.5 \%$ isoflurane anesthesia. The transducer was positioned in the aortic arch via the left carotid artery whilst the transmitter body was placed in a subcutaneous pocket in the right flank. Data were collected after a 7-day recovery period. Angiotensin II or saline was administered via osmotic minipumps (Model 1002, Alzet, Cupertino, CA) implanted subcutaneously under $2 \%$ isofurane.

\section{Western blotting}

Aortic samples were lysed using a polytron homogeniser in Tris $\mathrm{HCl}(25 \mathrm{mM})$ buffer solution $(\mathrm{pH} 7.2)$ containing EGTA ( $2 \mathrm{mM})$, EDTA $(5 \mathrm{mM}), \mathrm{NaF}(30 \mathrm{mM}), \beta$-glycerophosphate $(40 \mathrm{mM})$, sodium pyrophosphate $(20 \mathrm{mM})$, sodium orthovandate $(1 \mathrm{mM})$, phenylmethylsulfonyl-fluroide $(1 \mathrm{mM})$, benzamidine $(3 \mathrm{mM})$, pepstatin A $(5 \mu \mathrm{M})$, and leupeptin $(10 \mu \mathrm{M})$. Protein content was determined using a Biorad Bradford Protein assay (Biorad, UK). Proteins were separated by SDS-PAGE and transferred onto nitrocellulose membrane. The antibodies used were: Nox2, eNOS (BD Transduction Laboratories, UK); p22phox (gift from F.Wientjes, University College London, UK); phospho- and pan-ERK (Cell Signalling, MA); nitrotyrosine (Abcam); and actin (Sigma, UK). Immunoblots were quantified by densitometry, using the actin level for normalization. 


\section{Real-time RT-PCR}

RNA was isolated using an SV total RNA isolation kit (Promega, UK). cDNA was synthesized using avian myeloblastosis virus reverse transcriptase (AMV; Promega, UK) at $42^{\circ} \mathrm{C}$ for $90 \mathrm{~min}$. Relative gene expression was quantified on an Applied Biosystems 7000 sequence detection system (Applied Biosystems, UK) using SYBR Green and the comparative $\mathrm{Ct}$ method, with GADPH levels used for normalization. Forward and reverse primer sequences were as follow (all $5^{\prime}-3^{\prime}$ ):

GAPDH: CGTGCCGCCTGGAGAA, CCCTCAGATG CCTGCTTCAC; $\beta$-actin: GTGAAAAGATGACCCAGA TCA, TGGTACGACCAGAGGCATACAG; p22phox: TG GACGTTTCACACAGTGGT, AAAGAGGAAAAAGGG GTCCA; Nox1: CATCCAGTCTCCAAACATGACAG, GCTACAGTGGCAATCACTCCAGTA; Nox2: ACTCCT TGGGTCAGCACTGG, GTTCCTGTCCAGTTGTCTTCG; Nox4: TGAACTACAGTGAAGATTTCCTTGAAC, GAC ACCCGTCAGACCAGGAAT; p47phox: AGAGTCGCCA GGGCACTCT, TCTTCGCCTGGCTGTCAGT; p67phox: AAGCTGTTTGCCTGTGAGGT, CTTCATGTTGGTTGC CAATG; Catalase: GCTGAGAAGCCTAAGAACGCAAT, CCCTTCGCAGCCATGTG; SOD1: GGACCTCATTTTA ATCCTCACTCTAAG, GGTCTCCAACATGCCTCTCTTC; SOD2: CACACATTAACGCGCAGATCA, GGTGGCGTT GAGATTGTTCA; SOD3: GGGATGGATCTAGAGCATT AAGGA, ACACCTTAGTTAACCCAGAAATCTTTTC.

Immunohistochemistry and aortic morphology

Aortic sections for immunohistochemistry were frozen in OCT solution and stored at $-80^{\circ} \mathrm{C}$. Immunostaining was performed on $10 \mu \mathrm{m}$ cryosections. Sections were incubated with polyclonal anti-Nox2 (BD Transduction Laboratories) and anti-CD31 (Chemicon) antibodies. Secondary antibodies were conjugated to Alexafluor 488 or $\mathrm{Cy} 3$ and analyses were performed on a Leica SP5 confocal microscope (Leica, Germany). For aortic morphology, tissues were pressure fixed in vivo with $0.9 \%$ saline perfusion followed by $4 \%$ PFA and embedded in paraffin. 6- $\mu \mathrm{m}$ sections were stained with hematoxylin and eosin. Digital images were analyzed for aortic wall thickness using Volocity software (Volocity v5.0, Perkin Elmer, USA), measuring approximately 20 radial lines from the internal elastic lamina to the external lamina per section [8].

Superoxide dismutase (SOD) activity

Aortic homogenates were prepared in extraction buffer $\left(\mathrm{NaPO}_{4} 10\right.$ mM, EDTA 5 mM, EGTA 5 mM, NaCl 50 mM, Triton $1 \%$, protease inhibitor cocktail 1\% (Sigma), $\mathrm{pH} 7.8$ ) and separated by zymography on $12 \%$ non-denaturing
PAGE (Invitrogen, UK) in running buffer (Tris base $20 \mathrm{mM}$, glycine $150 \mathrm{mM}, \mathrm{pH} 8.5$ ). The gel was subsequently soaked in the dark in potassium phosphate (50 mM, pH 7.8) solution containing nitroblue tetrazolium (NBT, $275 \mu \mathrm{g} / \mathrm{ml})$, riboflavin $(65 \mu \mathrm{g} / \mathrm{ml})$ and $N, N, N 9, N 9$ tetramethyl-ethylenediamine (Temedm $3.2 \mu \mathrm{l} / \mathrm{ml}$ ). Bands were developed by exposing the gel to light whilst washing in $\mathrm{dH}_{2} \mathrm{O}$. The SOD isoforms [SOD1, Cu/Zn-SOD; SOD2, Mn-SOD; and SOD3, extracellular (ec)-SOD] were identified by their positions on the gel corresponding to their relative molecular weights and subsequently quantified by densitometry [6].

ROS measurements

NADPH oxidase activity was assessed by lucigeninenhanced chemiluminescence, as described previously [12]. Briefly, fresh aortae were incubated with or without angiotensin II $(1 \mu \mathrm{M})$ for $30 \mathrm{~min}$ in DMEM at $37^{\circ} \mathrm{C}$, prior to homogenization in a modified Krebs Henseleit solution ( $\mathrm{NaCl} 118 \mathrm{mM}, \mathrm{KCl} 4.7 \mathrm{mM}, \mathrm{CaCl}_{2} 2.5 \mathrm{mM}, \mathrm{NaHCO}_{3}$ $25 \mathrm{mM}, \quad \mathrm{KH}_{2} \mathrm{PO}_{4} 1.2 \mathrm{mM}, \mathrm{MgSO}_{4} 1.2 \mathrm{mM}$, glucose $11 \mathrm{mM}$, HEPES $10 \mathrm{mM}, \mathrm{pH}$ 7.4) containing protease inhibitor cocktail $(0.05 \mu \mathrm{l} / \mathrm{mg}$; Sigma). Tissue homogenates $(15 \mu \mathrm{g})$ were added to Krebs buffer solution, incubated at $37^{\circ} \mathrm{C}$ for $10 \mathrm{~min}$, followed by addition of NADPH $(300 \mu \mathrm{M})$ and dark-adapted lucigenin $(5 \mu \mathrm{M})$. Chemiluminescence was then measured on a plate luminometer (Lucy 1, Rosys Anthos, Wals, Austria) over a 20- to 30-min period. All samples were performed in triplicate and the level of superoxide production was calculated from average area under the curve. In some experiments, one of the following inhibitors was pre-incubated with the cell homogenate for $20 \mathrm{~min}$ prior to the addition of NADPH: the flavoprotein inhibitor, diphenyleneiodonium (DPI, $10 \mu \mathrm{M}$ ); a NOS inhibitor, $N$-nitro-L-arginine methyl ester hydrochloride (L-NAME, $100 \mu \mathrm{M}$ ); a mitochondrial electron transport chain inhibitor, rotenone $(50 \mu \mathrm{M})$; a superoxide scavenger, tiron $(10 \mathrm{mM})$; a putative NADPH oxidase inhibitor, apocynin $(10 \mu \mathrm{M})$; or a xanthine oxidase inhibitor, allopurinol $(100 \mu \mathrm{M})$.

Vascular tone

Segments of the descending thoracic aorta were suspended in an organ bath containing Krebs buffer solution comprising (in $\mathrm{mM}$ ) $\mathrm{NaCl} 118.2, \mathrm{KCl} 4.69, \mathrm{MgSO}_{4} \cdot 7 \mathrm{H}_{2} \mathrm{O} 1.18$, $\mathrm{KH}_{2} \mathrm{PO}_{4}$ 1.19, glucose 11.1, $\mathrm{NaHCO}_{3} 25.0, \mathrm{CaCl}_{2} \cdot 6 \mathrm{H}_{2} \mathrm{O}$ 2.5 and indomethacin $0.003 \mathrm{mM}, \mathrm{pH} 7.4$ at $37^{\circ} \mathrm{C}$, for isometric tension measurements. Rings were exposed to vasoconstrictors or vasodilators. For vasodilation studies, rings were pre-constricted to $75 \%$ of the maximal contraction to phenylephrine prior to addition of acetycholine 
or sodium nitroprusside. In some experiments, aortic rings were incubated with angiotensin II $(1 \mu \mathrm{M})$ for $30 \mathrm{~min}$ with or without tiron $(10 \mathrm{mM})$ prior to any other agents. In a different set of experiments, a single dose of $N$-methyl-Larginine acetate salt, (L-NMMA, $100 \mu \mathrm{M}$ ) was added to aortic rings pre-constricted to $30 \%$ of the maximal response to phenylephrine.

\section{Statistics}

Data are expressed as mean \pm SEM. Comparisons between Tg and wild-type were made by unpaired Student's $t$ test for two groups, one-way ANOVA with Bonferroni posthoc testing for more than two groups, or repeated measures ANOVA as appropriate. Two-way ANOVA was used to compare responses to treatments between $\mathrm{Tg}$ and wildtype. The entire concentration-response curves were compared by non-linear regression analysis followed by the extra sum-of-squares $F$ test. Statistical analyses were done on GraphPad Prism (v4.03 for Windows, San Diego, CA). $P<0.05$ was considered significant.

\section{Results}

Endothelial-specific overexpression of Nox2 in vivo

Two independent lines of endothelium-targeted Nox2overexpressing mice on a C57/B16 background were generated. The lines were similar and data presented herein are from line $1 . \mathrm{Tg}$ and WT mice were obtained in the predicted Mendelian ratio and there were no differences in gross morphology or in body and organ weights between genotypes (Online Resource 1). The level of Nox2 protein was significantly increased (twofold) in Tg aorta compared to wild-type (Fig. 1a) and was similarly increased in $\mathrm{Tg}$ line 2 (Online Resource 2A). Isolated cultured coronary microvascular endothelial cells (CMEC) of Tg also had a twofold increase in Nox2 levels compared to wild-type CMEC (Fig. 1b). Mechanical denudation of the endothelium from aortic segments reduced Nox2 proteins levels in both $\mathrm{Tg}$ and wild-type groups and abolished the difference between groups (Fig. 1c), indicating that the increase in Nox2 derived from the endothelium. The endothelialspecificity of Nox 2 overexpression was further confirmed by immunostaining sections of aorta, where an increase in Nox2 that co-localized with an endothelial marker, CD31, was observed in Tg (Fig. 2).

The effect of Nox 2 overexpression on NADPH oxidase activity was investigated in aortic homogenates. NADPHdependent superoxide production was similar in Tg compared to wild-type littermates (Fig. 3a). However, after acute exposure of aortae to angiotensin II $(0.1 \mu \mathrm{M}$, $30 \mathrm{~min}$ ), there was a significantly greater increase in NADPH oxidase activity in Tg compared to wild-type (85\% cf. 34\%; Fig. 3a). Diphenyleneiodonium (DPI, $10 \mu \mathrm{M})$, tiron $(10 \mathrm{mM})$ and apocynin $(10 \mu \mathrm{M})$ inhibited the chemiluminescence signal in all experiments, whereas L-NAME $(100 \mu \mathrm{M})$, rotenone $(2 \mu \mathrm{M})$ and allopurinol $(100 \mu \mathrm{M})$ had no effect (Online Resource 2), indicating that NADPH oxidase was the likely source. To also assess in vivo activation of Nox2, we studied aortic tissue from mice that were infused with saline or angiotensin II $(1.1 \mathrm{mg} / \mathrm{kg} /$ day for 2 weeks $)$. Aortic nitrotyrosine levels were quantified as a readout of increased NO/ROS interaction resulting from increased superoxide generation. Angiotensin II infusion increased nitrotyrosine levels in both groups of mice but the levels were significantly higher in the Tg group (Fig. 3b). These data are consistent with the knowledge that Nox 2 oxidase is normally quiescent and requires agonist activation so that an increase in expression level per se will not increase oxidase activity [1].
A

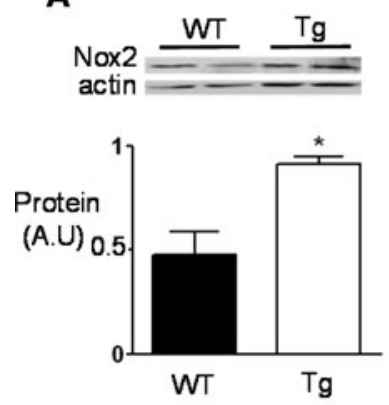

B
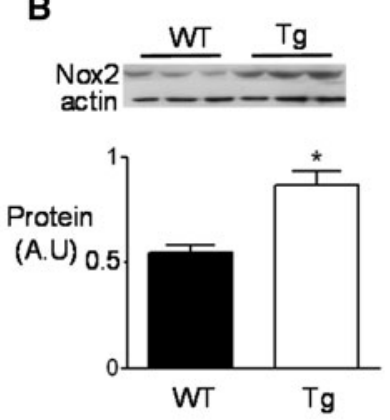
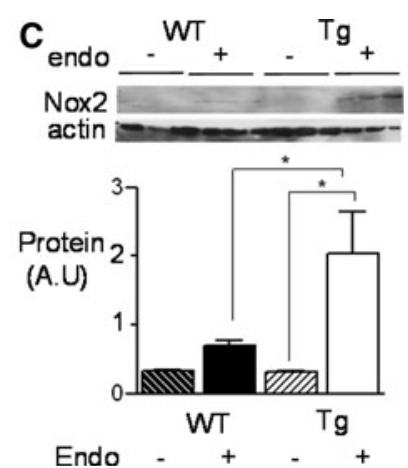

Fig. 1 Endothelial-specific Nox2 overexpression. Representative western blots and mean data showing Nox 2 protein level in a WT and $\mathrm{Tg}$ aorta and $\mathbf{b}$ isolated coronary microvascular endothelial cells. $n=6$ aortae/group, $n=5 \mathrm{CMEC}$ isolations/group, $* P<0.05$. c Nox 2 protein level in WT and Tg aorta with endothelium (endo) intact $(+)$ or removed $(-)$, as assessed by western blot. $n \geq 3$ per group, $* P<0.05$ 
Fig. 2 Immunostaining for Nox2. Aortic sections stained for Nox2 (green) and CD31 (red) as an endothelial marker. The yellow colour in the merged images in the bottom panels denotes co-localization. Scale bars $50 \mu \mathrm{m}$
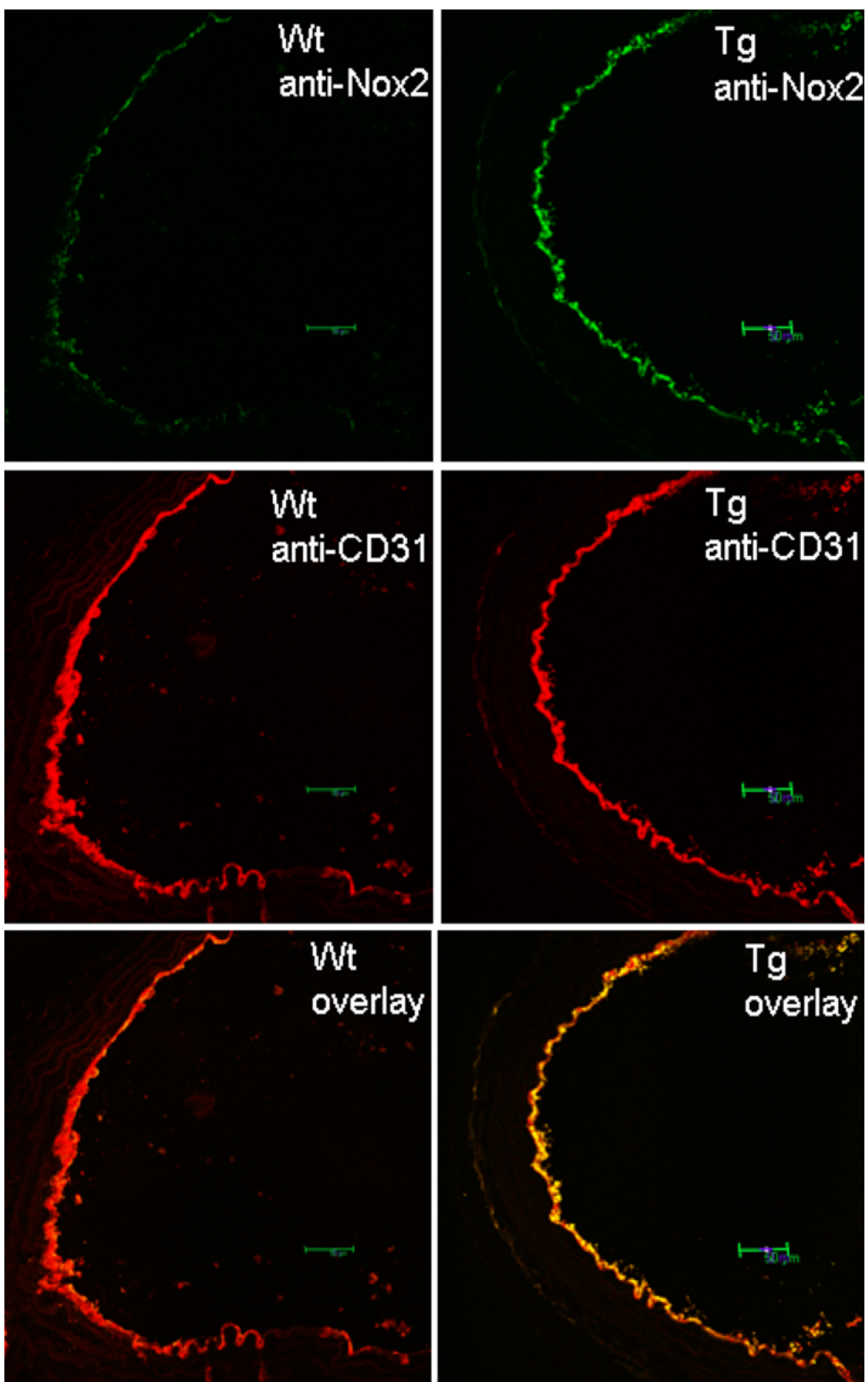

Effect of Nox2 overexpression on NADPH oxidase subunits, eNOS and antioxidants

We investigated whether overexpression of Nox 2 led to changes in other proteins required for Nox 2 activity. There were no differences in mRNA levels of p22phox, p47phox or p67phox between $\mathrm{Tg}$ and wild-type aorta (Fig. 3c). Because it is reported that p22phox and Nox2 proteins stabilize each other [2, 4, 18], we also measured the protein levels of p22phox. Indeed, this was found to be increased approximately threefold in the Tg group (Fig. 3d). Nox2 overexpression did not result in any changes in levels of Nox4, the main other Nox isoform expressed in the endothelium (Fig. 3c). Nox1 mRNA levels were below the level of detection in both groups (data not shown). There was no difference either in the mRNA levels of endothelial NO 
Fig. 3 Changes in NADPH oxidase activity, subunits and antioxidants. a NADPH oxidase activity in WT and Tg aortic homogenates under basal conditions (left) and after angiotensin II (AngII $0.1 \mu \mathrm{M}$, 30 min) stimulation (right). $n>10 ; P<0.05$.

b Nitrotyrosine levels in aorta from $\mathrm{Wt}$ and $\mathrm{Tg}$ mice treated with saline or angiotensin II infusion. Representative blots and mean data from three experiments $(* P<0.05)$. c mRNA expression of p22phox, p47phox, p67phox, Nox4 and eNOS in aorta. $N=6, P=$ NS. d Protein expression of $\mathrm{p} 22$ phox in aorta. Representative blots at top and mean data from three experiments at bottom. $* P<0.05$. e mRNA expression of SOD isoforms and catalase in aorta. $n>6, * P<0.05$. f SOD activity by NBT zymography in aortic homogenates. $N=6$, $P=\mathrm{NS}$
A
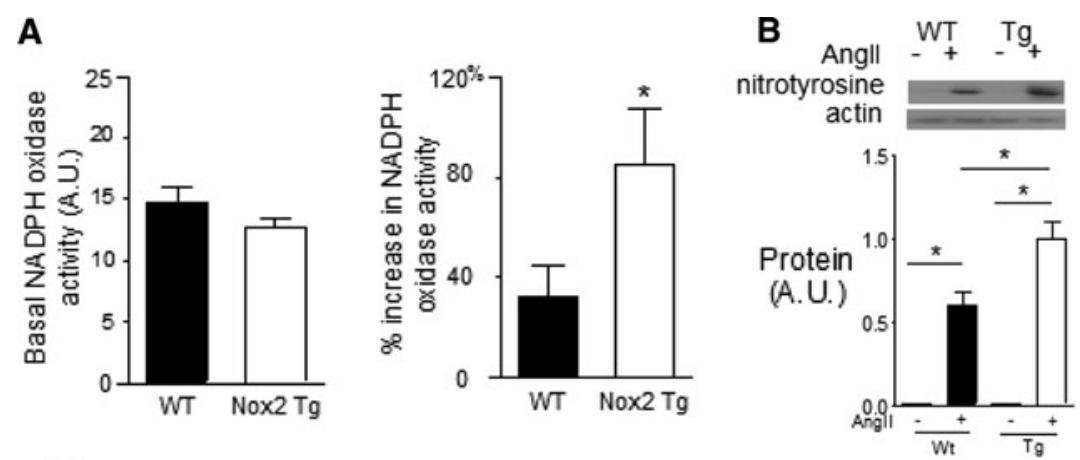

C

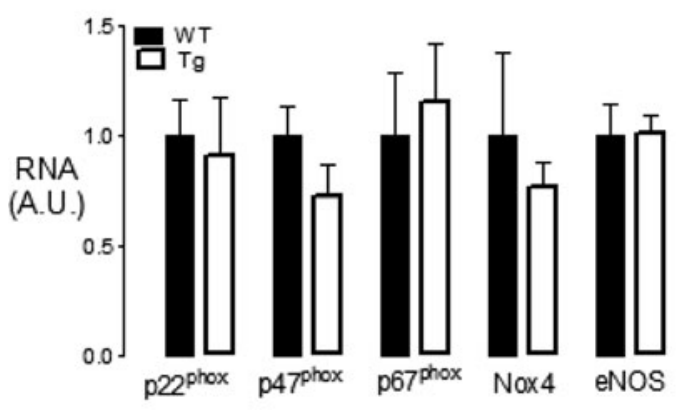

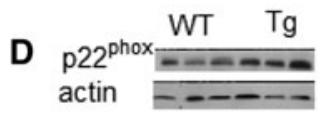

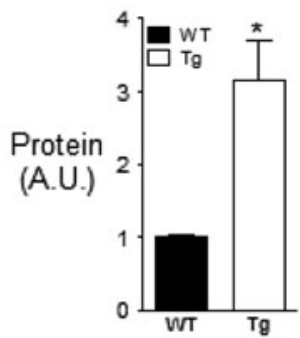

E

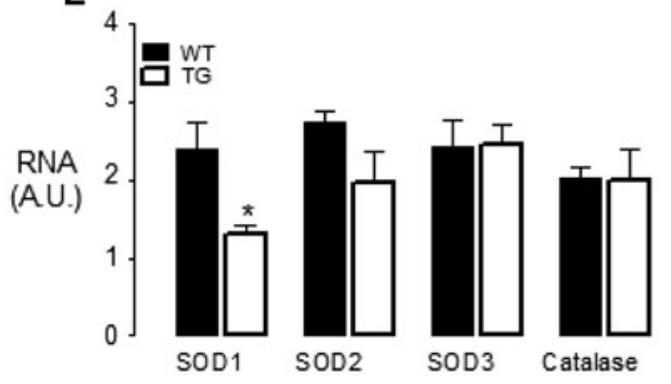

$\mathbf{F}$

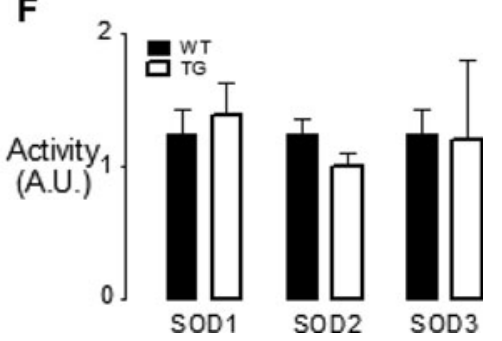

synthase (eNOS) between $\mathrm{Tg}$ and wild-type groups (Fig. 3c) or in the eNOS protein levels (Online Resource 2C).

Since the effects of ROS generated by NADPH oxidase may be influenced by ambient levels of antioxidant enzymes, in particular superoxide dismutases (SODs) and catalase, we also assessed these. There was a slight but significant reduction in SOD1 mRNA levels in Tg compared to wild-type but no difference in levels of SOD2, SOD3 and catalase (Fig. 3e). However, SOD1 activity was unaltered in Tg compared to wild-type as were SOD2 and SOD3 activities (Fig. 3f). Taken together, these data indicate that a modest overexpression of Nox 2 which does not alter basal NADPH oxidase activity does not induce significant changes in antioxidant genes or eNOS. Antioxidant levels were also assessed in aorta from mice that had been infused with angiotensin II ( $1.1 \mathrm{mg} / \mathrm{kg} /$ day for 2 weeks). Whereas angiotensin II infusion increased the expression of SOD1, SOD2 and catalase, the levels remained similar between wild-type and Tg groups (Online resource 3).
Effect of Nox2 overexpression on vascular tone

To determine the effect of increased endothelial Nox2 levels on vasomotor tone, we studied isolated aortic rings from $\mathrm{Tg}$ and wild-type littermate mice. Constrictor responses to potassium chloride $(80 \mathrm{mM})$ and phenylephrine were similar between groups (Fig. $4 \mathrm{a}, \mathrm{b}$ ). Endothelium-dependent vasorelaxation to acetylcholine and endothelial-independent relaxation to sodium nitroprusside were both similar in wild-type and Tg groups (Fig. 4c, d). To study vasorelaxation under conditions where Nox 2 was activated, aortic rings were stimulated with angiotensin II $(1 \mu \mathrm{M}, 30 \mathrm{~min})$ prior to the assessment of dilator responses. A small but significant decrease in the maximum acetylcholine-induced relaxation was observed in angiotensin II-treated wild-type rings compared to saline control when comparing the entire curve but there was no change in $\mathrm{EC}_{50}\left(\log \mathrm{EC}_{50}:-7.37 \pm 0.08\right.$ vs. $-7.46 \pm 0.04 \mathrm{M}$, respectively, $P=\mathrm{ns} ; n=10$ ) (Fig. $4 \mathrm{e}$ ). The angiotensin II-treated $\mathrm{Tg}$ group showed a greater reduction in 
Fig. 4 Endothelial function in isolated aortae from Tg and WT mice. a Aortic contraction to a single dose of $\mathrm{KCl}(80 \mathrm{mM})$.

$N=10$. b Dose response curve to phenylephrine (PE; $10^{-9}$ to $\left.10^{-6} \mathrm{M}\right) . N=10$.

c Endothelial-dependent relaxation to acetylcholine (ACh). $N=10$. d Relaxation to sodium nitroprusside (SNP).

$N=10$. e, f Relaxation to Ach in the presence and absence of AngII $(0.1 \mu \mathrm{M}, 30 \mathrm{~min})$ in WT and $\mathrm{Tg}$ aorta, respectively. $N=10 .{ }^{*} P<0.05$ comparing $\mathrm{EC}_{50}$ and $E_{\max } \cdot \mathbf{g}, \mathbf{h}$ Relaxation to SNP in WT and $\mathrm{Tg}$,

respectively, in the presence and absence of AngII $(0.1 \mu \mathrm{M}$, $30 \mathrm{~min}$ ). $N=10$. i, j Relaxation to ACh in WT and Tg aortae, respectively, after co-incubation with AngII $(0.1 \mu \mathrm{M}, 30 \mathrm{~min})$ and the superoxide scavenger, tiron (10 mM). $N=3, P=\mathrm{NS}$
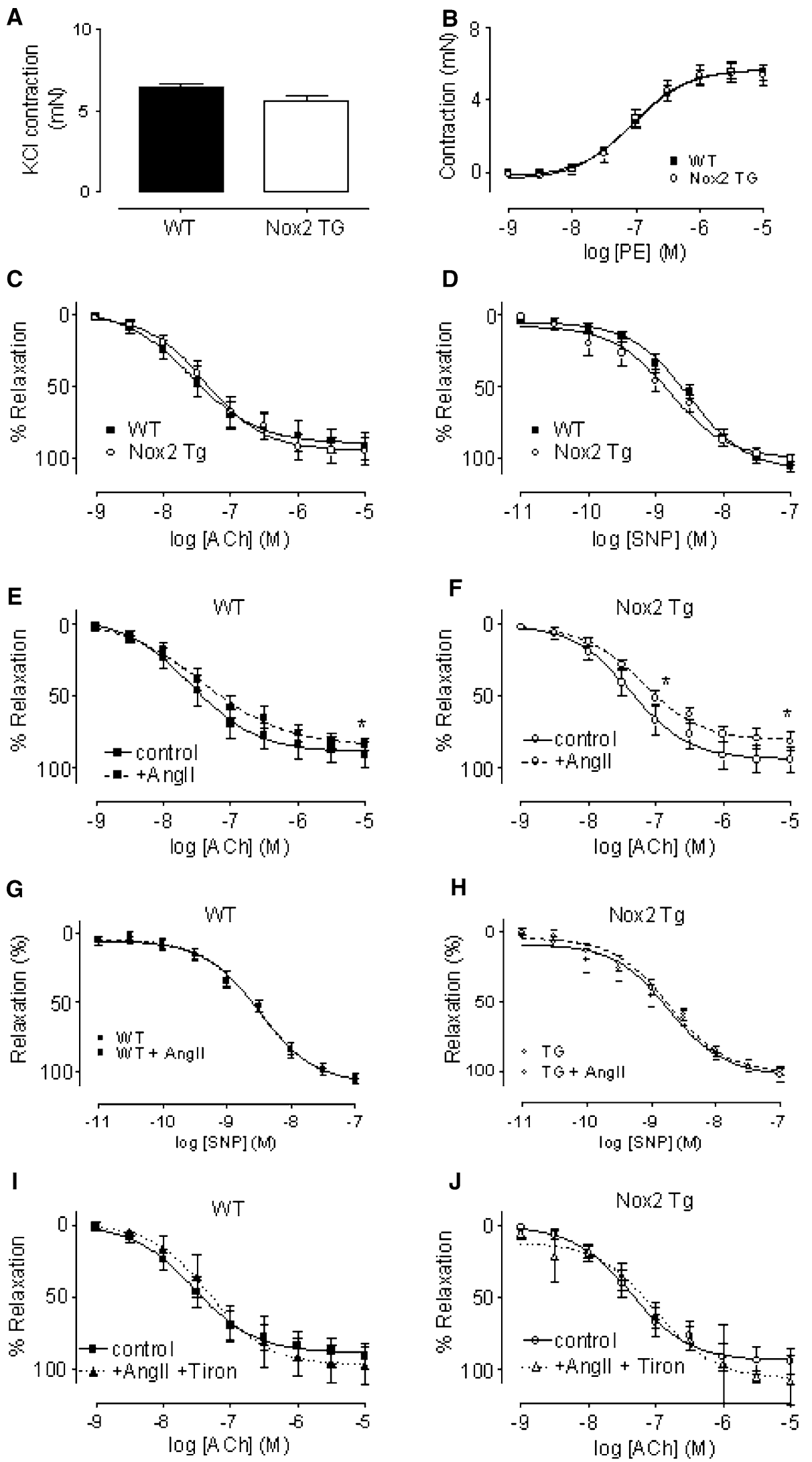
acetylcholine-induced relaxation with a modest but significant rightward shift of the EC50 $\left(\operatorname{logEC} \mathrm{C}_{50}\right.$ : $-7.19 \pm 0.05$ vs. $-7.37 \pm 0.05 \mathrm{M}$, respectively, $* P<0.05$; $n=10$ ) (Fig. 4f). Angiotensin II administration had no effect on endothelial-independent relaxation to sodium nitroprusside in either group (Fig. 4g, h). Pre-treatment with the superoxide scavenger, tiron $(10 \mathrm{mM})$ prior to angiotensin II treatment prevented the angiotensin IIinduced attenuation of the acetylcholine relaxation in both Wt and Tg aorta (Fig. 4i, j). The level of basal NO formation was assessed by studying the response to the NOS inhibitor, L-NMMA, in lightly pre-constricted aortic rings (30\% of phenylephrine maximum). The response in $\mathrm{Wt}$ and $\mathrm{Tg}$ vessels was found to be similar (3.9 $\pm 0.7 \mathrm{vs}$. $3.4 \pm 0.6 \mathrm{mN}$, respectively, $P=\mathrm{ns} ; n=3$ ).

Effect of Nox 2 overexpression on blood pressure and the in vivo response to chronic angiotensin II infusion

Ambulatory blood pressure was measured in $\mathrm{Tg}$ and wildtype mice by telemetry. There was no difference in systolic, diastolic or mean blood pressure between groups at baseline (Fig. 5a). To assess the response to angiotensin II, mice were implanted with osmotic minipumps infusing either angiotensin II or saline over a 2-week period. Saline infusion did not cause any changes in blood pressure (Fig. 5a). A low dose of angiotensin II (i.e. $0.3 \mathrm{mg} / \mathrm{kg} /$ day), which normally has no effect on blood pressure in wildtype mice caused significant increases in systolic, diastolic and mean blood pressure in Tg mice but no change in wildtype littermates (Fig. 4b). We also tested the effects of a high dose of angiotensin II (1.1 mg/kg/day). This resulted in similar rises in systolic and mean blood pressure in $\mathrm{Tg}$ and wild-type mice but diastolic blood pressure increased to a significantly greater extent in $\mathrm{Tg}$ compared with wildtype (Fig. 5c).Heart rates (Fig. 5, lower panels) and activity level (not shown) were similar in the $\mathrm{Tg}$ and wildtype groups).

\section{Aortic remodeling}

To assess whether the vasculature of $\mathrm{Tg}$ and wild-type mice had undergone angiotensin II-induced structural remodeling, we quantified medial area in aortic sections obtained from animals subjected to angiotensin II or saline infusion. A similar increase in medial area was found in $\mathrm{Tg}$ and wild-type mice subjected to low dose angiotensin II infusion ( $0.3 \mathrm{mg} / \mathrm{kg} /$ day) (Fig. 6a). With the higher dose infusion of angiotensin II $(1.1 \mathrm{mg} / \mathrm{kg} /$ day), however, aortic medial thickness was significantly greater in Tg compared to wild-type (Fig. 6a). The mean data for these experiments are shown in Fig. 6b. Finally, we investigated the effect of Nox 2 overexpression on the activation of ERK1, which may be involved in angiotensin II-induced structural remodeling. The protein levels of phospho-ERK were similar in unstimulated wild-type and $\mathrm{Tg}$ aorta but in angiotensin II-treated aorta, the levels of phospho-ERK were significantly greater in the $\mathrm{Tg}$ group (Fig. 6c).

\section{Discussion}

In this study, we have investigated the effects of endothelial-targeted in vivo overexpression of the Nox 2 isoform of NADPH oxidase on vascular function and angiotensin II-induced hypertension. Our main findings were that: (1) a modest twofold increase in levels of endothelial Nox 2 had no effect on basal NADPH oxidase activity, vascular function or blood pressure in the absence of agonist stimulation, consistent with the knowledge that this isoform is quiescent unless stimulated [1, 2, 4, 18]; (2) there were no significant compensatory changes in mRNA levels of Nox4, eNOS, or catalase or in SOD1-3 activity after Nox2 overexpression; (3) exposure to angiotensin II resulted in a significantly greater worsening of endothelial vasodilator function in $\mathrm{Tg}$ mice compared to wild-type but the magnitude of effect was very modest; (4) angiotensin II-induced hypertension was potentiated in $\mathrm{Tg}$ mice after chronic infusion of either a low (normally subpressor) dose or a high dose of angiotensin II; and (5) chronic high dose angiotensin II infusion caused greater vascular remodeling in $\mathrm{Tg}$ mice compared to wild-type. Taken together, these results suggest that an increase in endothelial levels of Nox 2 contributes significantly to hypertension in settings where there is increased activation of the renin-angiotensin system, an effect that may involve endothelial dysfunction and/or vascular structural remodeling.

Angiotensin II is well known to play a major role in the initiation and progression of hypertension as well as other vascular diseases [21]. Whilst the signal transduction of angiotensin II responses is complex, a large body of evidence points to an important role of ROS generated by NADPH oxidases [15, 18, 21, 24]. Chronically elevated levels of angiotensin II increase NADPH oxidase activity and superoxide production in all layers of the vessel wall but the relative contribution of different Nox isoforms and different cell types to the development of hypertension remains incompletely defined. As mentioned earlier, studies in gene-modified mouse models support an important role for VSMC Nox1 in angiotensin II-dependent hypertension [8, 11, 13, 20]. Expression levels of Nox2 increase upon angiotensin II stimulation in endothelial cells in vitro [25] as well as in the endothelium in situ in blood vessels [17] but the role of Nox 2 in the endothelium - a major site 
Fig. 5 Effect of chronic angiotensin II treatment on blood pressure in $\mathrm{Tg}$ and $\mathrm{WT}$ mice. Ambulatory blood pressure was measured in WT and $\mathrm{Tg}$ mice before and after implantation of osmotic pumps delivering either a saline, b low dose AngII $(0.3 \mathrm{mg} / \mathrm{kg} /$ day $)$ or c high dose AngII $(1.1 \mathrm{mg} / \mathrm{kg} /$ day). Systolic, diastolic and mean blood pressure and heart rate were averaged over $24 \mathrm{~h}$ periods. $n=6, * P<0.05$ for a significant interaction by twoway ANOVA
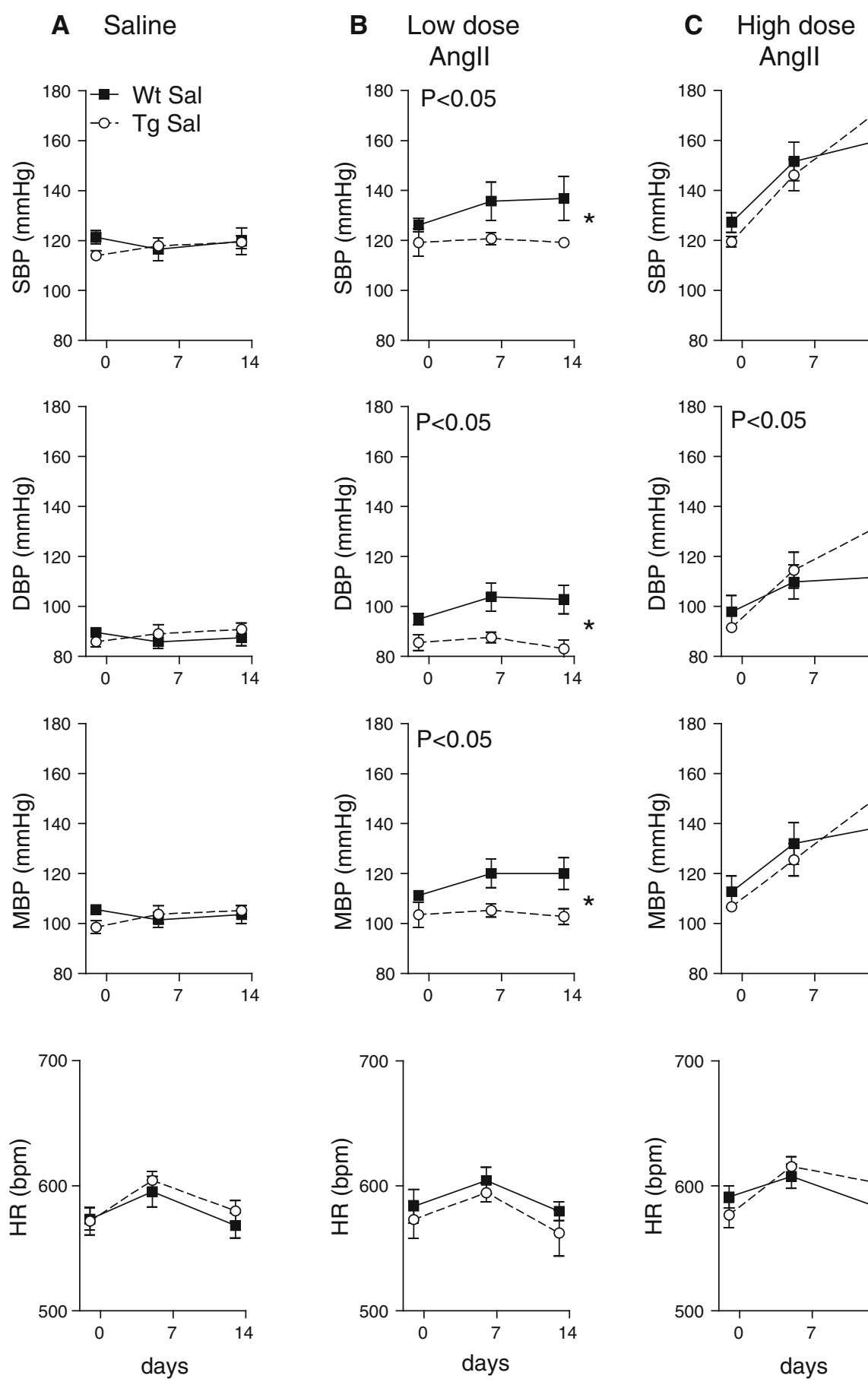
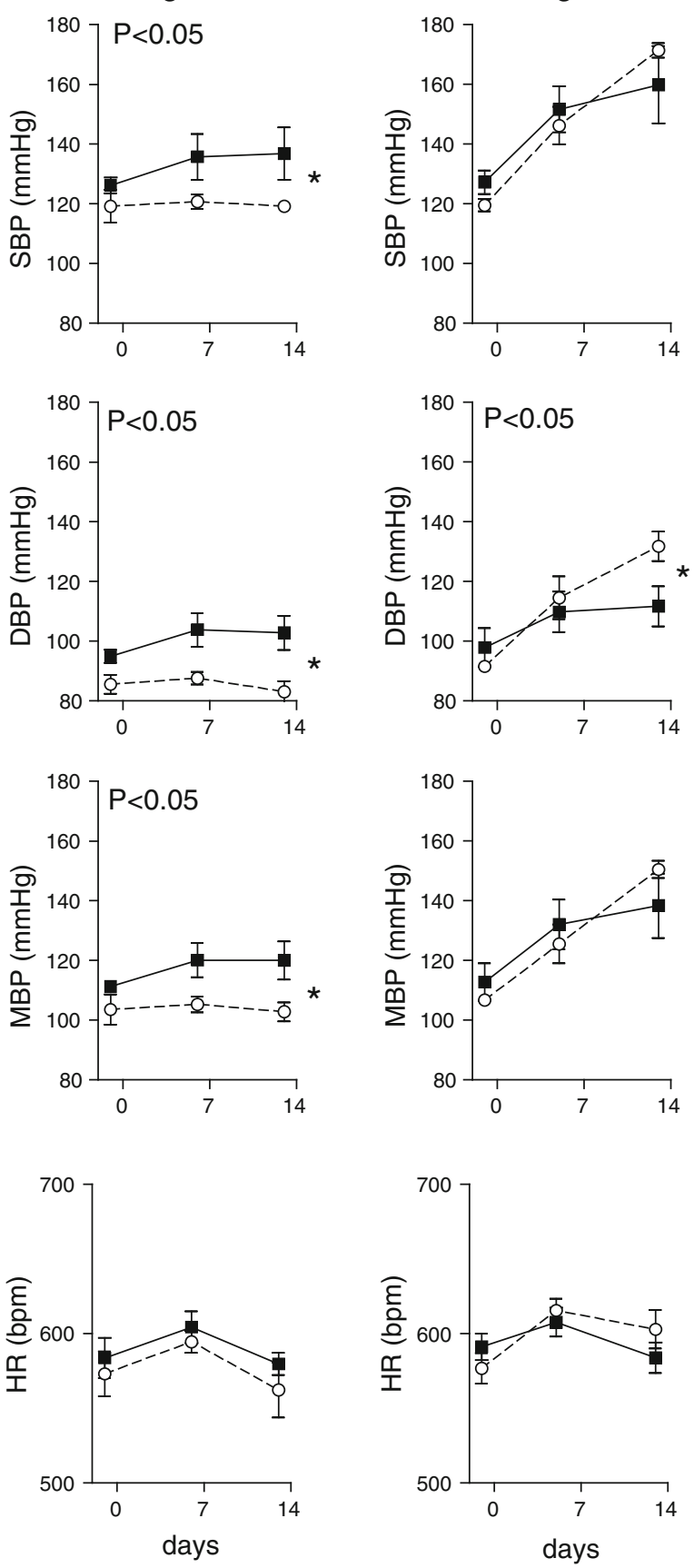

of expression of this isoform-in the development of hypertension is unclear.

In the current study, we undertook a specific endothelium-targeted overexpression of Nox2 using the well-established tie 2 promoter construct [26]. Endothelial-specificity of overexpression was confirmed by several approaches including immunostaining and the quantification of Nox2 levels in isolated endothelial cells and in aorta with or without endothelium. The degree of Nox2 expression that was achieved in $\mathrm{Tg}$ mice was comparable with the levels that have been reported in experimental models of endothelial dysfunction or hypertension [8, 11], suggesting that the data may be pathophysiologically relevant. Importantly, the basal endothelial-specific increase in Nox2 levels was not accompanied by potentially confounding changes in levels of Nox4, antioxidant genes or eNOS. Previous studies in which p22phox was overexpressed in VMSC in vivo were accompanied by numerous 

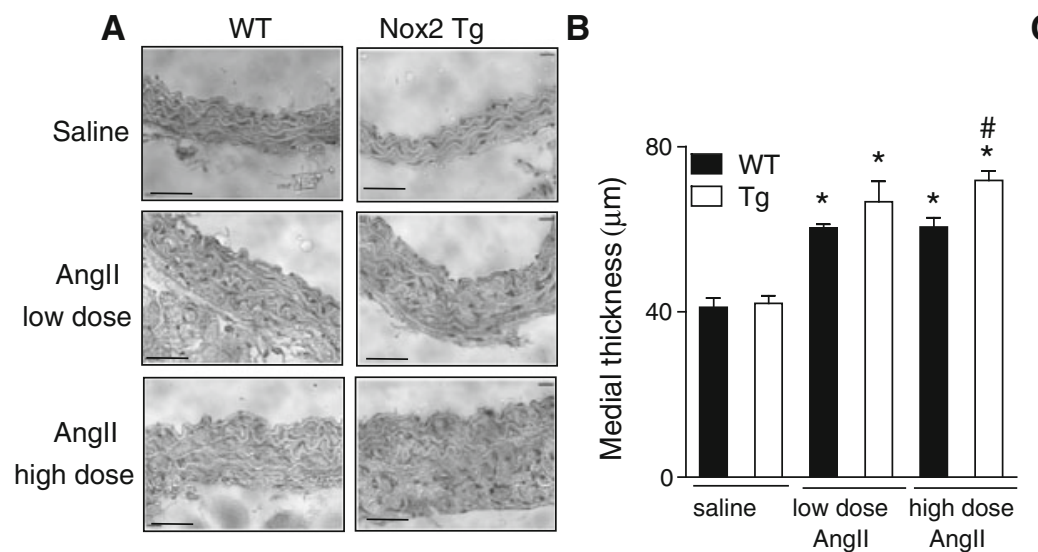

C P-ERK

pan-ERK

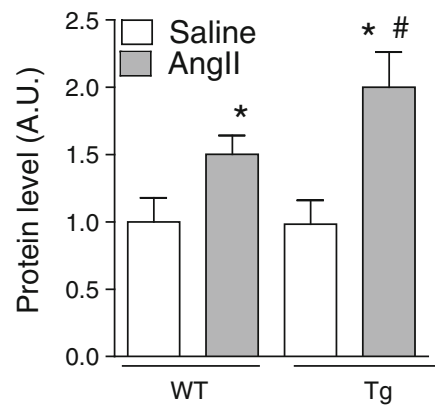

Fig. 6 Aortic hypertrophy and ERK signaling. a Representative images of H\&E-stained aortic sections from WT and Tg mice treated with infusion of saline, low dose AngII or high dose AngII. Scale bars $50 \mu \mathrm{m}$. b Mean data for aortic medial thickness from experiments illustrated in a. ${ }^{*} P<0.05$ sal versus AngII, ${ }^{\#} P<0.05$ WT AngII versus Tg AngII; two-way ANOVA; $n=5$ per group. c Levels of
ERK1/2 phosphorylation in WT and Tg aorta after 14-day treatment with saline or high dose AngII. Representative blots are shown at the top and mean data at the bottom. p-ERK1/2, phospho-ERK1/2; panERK1/2, total ERK1/2. $n=4,{ }^{*} P<0.05$ sal versus AngII, ${ }^{\#} P<0.05$ WT AngII versus Tg AngII

by a significant but rather modest worsening of endothelium-dependent vasodilatation. The most likely explanation for the modest impact of endothelial Nox2 on endothelial function is that ROS generated in all layers of the vessel wall during angiotensin II stimulation can interact with NO so that the relative impact of endothelial Nox2-derived ROS may be quite small. We also found significant differences in angiotensin II-induced hypertension in vivo. Using the gold-standard method of ambulatory telemetry, we found that there was no difference in blood pressure between $\mathrm{Tg}$ mice and wild-type littermates at baseline (consistent with the lack of increase in basal NADPH oxidase activity) but that a low dose chronic infusion of angiotensin II that is subpressor in wild-type mice significantly enhanced both diastolic and systolic blood pressures in Tg mice. These findings are significant as they suggest that a modest increase in endothelial Nox2 activity can lead to significant hypertension. Whereas previous studies have focused on VSMC Nox1 as being pivotal for the development of AngII-induced hypertension [8, 11, 13, 20], the current study suggests that endothelial Nox 2 could also contribute. Interestingly, at a higher dose of angiotensin II, we found that only diastolic but not systolic pressure was augmented in Tg mice. A previous study by Bendall et al. [3] in a similar model of endothelial-specific Nox2 overexpression also reported an increased systolic blood pressure response to low dose angiotensin II infusion as measured by tail-cuff plethysmography. However, that study did not study the responses to high dose angiotensin II nor were changes in diastolic blood pressure (which cannot be accurately quantified by tail-cuff plethysmography) reported. The difference between systolic and diastolic blood pressure responses to high dose angiotensin II 
infusion could reflect a predominant effect of endothelial Nox 2 on resistance vessels, since it is known that resistance vessel remodeling or increased tone can be an important driver of diastolic hypertension. On the other hand, systolic hypertension in this setting may be driven by mechanisms independent of endothelial Nox2-such as other Noxs or ROS-independent mechanisms [22, 23]. Several ROSdependent mechanisms can potentially contribute to the development of angiotensin II-dependent hypertension. Although the magnitude of difference in endotheliumdependent relaxation between $\mathrm{Tg}$ and wild-type assessed in aortic strips in vitro was quite modest, this could nevertheless be a contributor in vivo. The relevance of this mechanism would ideally require the assessment of resistance vessel tone and remodeling in vessels from animals subjected to angiotensin II infusion. Although vessel tone or NO production was not investigated in the mice treated with angiotensin II infusion, we were able to demonstrate evidence of enhanced $\mathrm{NO} /$ superoxide interaction in the vessels of Tg animals-as assessed by nitrotyrosine levels. We also assessed vascular structural remodeling in the aorta and found that aortic medial area was greater after high-dose but not low dose angiotensin II in the Tg mice. A potential mechanism contributing to the increased remodeling after high dose angiotensin II could be an increased activation of ERK signaling after AngII infusion in Tg mice compared to Wt. The lack of observed difference in aortic medial area in the low dose angiotensin II group suggests that other mechanisms (such as altered endothelial function) were important. It is also possible that altered endothelial function in renal and/or central nervous system vessels leads to perturbation of function that drives hypertension [22, 23]. Additional studies will be required to distinguish between these possibilities.

It is well documented that ROS generation and, in particular, NADPH oxidase are important components involved in angiotensin II-induced hypertension [2, 4, 18, 21]. The recognition of differential expression of the multiple Nox isoforms in the vessel wall, their varying regulation, and their differential expression in disease settings make it necessary to assess the contributions of individual isoforms in different locations. The current study shows that increases in endothelial Nox2 levels may contribute significantly to the development of angiotensin II-induced hypertension through mechanisms that may include alterations in endothelial vasodilator function and/ or an enhancement of vascular structural remodeling.

Acknowledgments This work was supported by the British Heart Foundation (BHF), a Fondation Leducq Transatlantic Network of Excellence award, and in part by EU FP6 Grant LSHM-CT-2005018833, EUGeneHeart. AMS holds the BHF Chair of Cardiology at King's College London. CM and SAR were funded by BHF PhD studentships.
Open Access This article is distributed under the terms of the Creative Commons Attribution Noncommercial License which permits any noncommercial use, distribution, and reproduction in any medium, provided the original author(s) and source are credited.

\section{References}

1. Anilkumar N, Weber R, Zhang M, Brewer A, Shah AM (2008) Nox 4 and Nox 2 NADPH oxidases mediate distinct cellular redox signaling responses to agonist stimulation. Arterioscler Thromb Vasc Biol 28:1347-1354. doi:10.1161/ATVBAHA.108.164277

2. Bedard K, Krause KH (2007) The NOX family of ROS-generating NADPH oxidases: physiology and pathophysiology. Physiol Rev 87:245-313. doi:10.1152/physrev.00044.2005

3. Bendall JK, Rinze R, Adlam D, Tatham AL, de Bono J, Channon KM (2007) Endothelial Nox 2 overexpression potentiates vascular oxidative stress and hemodynamic response to angiotensin II: studies in endothelial-targeted Nox 2 transgenic mice. Circ Res 100:1016-1025. doi:10.1161/01.RES.0000263381.83835.7b

4. Brandes RP, Weissmann N, Schröder K (2010) NADPH oxidases in cardiovascular disease. Free Radic Biol Med 49:687-706. doi: 10.1016/j.freeradbiomed.2010.04.030

5. Byrne JA, Grieve DJ, Bendall JK, Li JM, Gove C, Lambeth JD, Cave AC, Shah AM (2003) Contrasting roles of NADPH oxidase isoforms in pressure-overload versus angiotensin II-induced cardiac hypertrophy. Circ Res 93:802-805. doi:10.1161/01.RES. 0000099504.30207.F5

6. Culotta VC, Klomp LWJ, Strain J, Casareno RL, Krems B, Gitlin JD (1997) The copper chaperone for superoxide dismutase. J Biol Chem 272:23469-23472

7. Delles C, Miller WH, Dominiczak AF (2008) Targeting reactive oxygen species in hypertension. Antioxid Redox Signal 10:1061-1078. doi:10.1089/ars.2007.2008

8. Dikalova A, Clempus R, Lassegue B, Cheng G, McCoy J, Dikalov S, San MA, Lyle A, Weber DS, Weiss D, Taylor WR, Schmidt HH, Owens GK, Lambeth JD, Griendling KK (2005) Nox 1 overexpression potentiates angiotensin II-induced hypertension and vascular smooth muscle hypertrophy in transgenic mice. Circulation 112:2668-2676. doi:10.1161/CIRCULATIONAHA. 105.538934

9. Dworakowski R, Walker S, Momin A, Desai J, El-Gamel A, Wendler O, Kearney MT, Shah AM (2008) Reduced nicotinamide adenine dinucleotide phosphate oxidase-derived superoxide and vascular endothelial dysfunction in human heart failure. J Am Coll Cardiol 51:1349-1356. doi:10.1016/j.jacc.2007.12.033

10. Guzik TJ, West NE, Black E, McDonald D, Ratnatunga C, Pillai R, Channon KM (2000) Vascular superoxide production by $\mathrm{NAD}(\mathrm{P}) \mathrm{H}$ oxidase: association with endothelial dysfunction and clinical risk factors. Circ Res 86:E85-E90

11. Gavazzi G, Banfi B, Deffert C, Fiette L, Schappi M, Herrmann F, Krause KH (2006) Decreased blood pressure in NOX1-deficient mice. FEBS Lett 580:497-504. doi:10.1016/j.febslet.2005.12.049

12. Grieve DJ, Byrne JA, Siva A, Layland J, Johar S, Cave AC, Shah AM (2006) Involvement of the nicotinamide adenosine dinucleotide phosphate oxidase isoform Nox2 in cardiac contractile dysfunction occurring in response to pressure overload. J Am Coll Cardiol 47:817-826. doi:10.1016/j.jacc.2005.09.051

13. Hassanain HH, Gregg D, Marcelo ML, Zweier JL, Souza HP, Selvakumar B, Ma Q, Moustafa-Bayoumi M, Binkley PF, Flavahan NA, Morris M, Dong C, Goldschmidt-Clermont PJ (2007) Hypertension caused by transgenic overexpression of Rac1. Antioxid Redox Signal 9:91-100. doi:10.1089/ars.2007.9.91 
14. Heusch G, Schulz R (2011) A radical view on the contractile machinery in human heart failure. J Am Coll Cardiol 57:310-312. doi:10.1016/j.jacc.2010.06.057

15. Iliodromitis EK, Andreadou I, Prokovas E, Zoga A, Farmakis D, Fotopoulou T, Ioannidis K, Paraskevaidis IA, Kremastinos DT (2010) Simvastatin in contrast to postconditioning reduces infarct size in hyperlipidemic rabbits: possible role of oxidative/nitrosative stress attenuation. Basic Res Cardiol 105:193-203. doi: 10.1007/s00395-009-0078-3

16. Johar S, Cave AC, Narayanapanicker A, Grieve DJ, Shah AM (2006) Aldosterone mediates angiotensin II-induced interstitial cardiac fibrosis via a Nox2-containing NADPH oxidase. FASEB J 20:1546-1548. doi:10.1096/fj.05-4642fje

17. Jung O, Schreiber JG, Geiger H, Pedrazzini T, Busse R, Brandes RP (2004) gp91phox-containing NADPH oxidase mediates endothelial dysfunction in renovascular hypertension. Circulation 109:1795-1801. doi:10.1161/01.CIR.0000124223.00113.A4

18. Lambeth JD (2004) NOX enzymes and the biology of reactive oxygen. Nat Rev Immunol 4:181-189. doi:10.1038/nri1312

19. Laude K, Cai H, Fink B, Hoch N, Weber DS, McCann L, Kojda G, Fukai T, Schmidt HH, Dikalov S, Ramasamy S, Gamez G, Griendling KK, Harrison DG (2005) Hemodynamic and biochemical adaptations to vascular smooth muscle overexpression of p22phox in mice. Am J Physiol 288:H7-H12. doi:10.1152/ ajpheart.00637.2004

20. Matsuno K, Yamada H, Iwata K, Jin D, Katsuyama M, Matsuki M, Takai S, Yamanishi K, Miyazaki M, Matsubara H, YabeNishimura C (2005) Nox1 is involved in angiotensin II-mediated hypertension: a study in Nox1-deficient mice. Circulation 112:2677-2685. doi:10.1161/CIRCULATIONAHA.105.573709

21. Mehta PK, Griendling KK (2007) Angiotensin II cell signaling: physiological and pathological effects in the cardiovascular system. Am J Physiol 292:C82-C97. doi:10.1152/ajpcell. 00287.2006

22. Peterson J, Sharma R, Davisson R (2006) Reactive oxygen species in the neuropathogenesis of hypertension. Curr Hypertens Rep 8:232-241

23. Ponnuchamy B, Khalil RA (2009) Cellular mediators of renal vascular dysfunction in hypertension. Am J Physiol 296:R1001R1018. doi:10.1152/ajpregu.90960.2008

24. Pöss J, Werner C, Lorenz D, Gensch C, Böhm M, Laufs U (2010) The renin inhibitor aliskiren upregulates pro-angiogenic cells and reduces atherogenesis in mice. Basic Res Cardiol 105:725-735. doi:10.1007/s00395-010-0120-5

25. Rueckschloss U, Quinn MT, Holtz J, Morawietz H (2002) Dosedependent regulation of $\mathrm{NAD}(\mathrm{P}) \mathrm{H}$ oxidase expression by angiotensin II in human endothelial cells: protective effect of angiotensin II type 1 receptor blockade in patients with coronary artery disease. Arterioscler Thromb Vasc Biol 22:1845-1851

26. Schlaeger TM, Bartunkova S, Lawitts JA, Teichmann G, Risau W, Deutsch U, Sato TN (1997) Uniform vascular-endothelialcell-specific gene expression in both embryonic and adult transgenic mice. Proc Natl Acad Sci USA 94:3058-3063

27. Tiyerili V, Zimmer S, Jung S, Wassmann K, Naehle CP, Lütjohann D, Zimmer A, Nickenig G, Wassmann S (2010) CB1 receptor inhibition leads to decreased vascular AT1 receptor expression, inhibition of oxidative stress and improved endothelial function. Basic Res Cardiol 105:465-477. doi:10.1007/ s00395-010-0090-7

28. Touyz RM, Mercure C, He Y, Javeshghani D, Yao G, Callera GE, Yogi A, Lochard N, Reudelhuber TL (2005) Angiotensin IIdependent chronic hypertension and cardiac hypertrophy are unaffected by gp91phox-containing NADPH oxidase. Hypertension 45:530-537. doi:10.1161/01.HYP.0000158845.49943.5e 